trol through covalent conjugation of biomolecules, which can be accomplished by applying surface chemical functionality, such as carboxyls or amines, on the silica nanoparticles using well-established silane coupling chemistry."

STEVEN TROHALAKI

\section{Single-Particle Electrode Microbatteries Studied}

Because the factors limiting the performance of Li-based batteries are not well known, research groups have begun to use micron-sized single-particle electrodes to improve their understanding of the performance of electrode materials relevant to both aqueous and nonaqueous battery systems. A group from the Department of Chemistry at Case Western University has taken these studies a step further by assembling "microbatteries" from singleparticle cathodes and anodes.

In the February issue of Electrochemical and Solid-State Letters (p. A122), researchers Q. Shi and D.A. Scherson of Case Western University describe their fabrication of single-particle electrode microbatteries by placing individual microparticles of cathode and anode materials on isolated microelectrodes. They chose $\mathrm{LiMn}_{2} \mathrm{O}_{4}$ on Pt as the cathode, and a carbon particle on $\mathrm{Ni}$ was used for the anode. Both electrode particles were $\sim 50 \mu \mathrm{m}$ in diameter and separated by a distance of $\sim 80 \mu \mathrm{m}$. The researchers employed $1 \mathrm{M}$ solutions of $\mathrm{LiClO}_{4}$ and $\mathrm{LiPF}_{6}$ as electrolytes, and the entire composite structure was sealed in an electrochemical cell.

The researchers focused their first set of experiments on isolated microelectrodes. $\mathrm{LiMn}_{2} \mathrm{O}_{4}$ cathodes were charged fully, and thereafter, their discharge or open-circuit potential versus time characteristics were measured. When $\mathrm{LiClO}_{4}$ was chosen as the electrolyte, a rapid stepwise discharge was observed. Plateaus in potential were observed between $0-2 \mathrm{~h}$, then between 2-8 h, after which discharge occurred quickly. A significant improvement was achieved using $\mathrm{LiPF}_{6}$ as the electrode, as the potential quickly stabilized at $4.1 \mathrm{~V}$ and remained stable for over $35 \mathrm{~h}$. Importantly, the researchers said, this outcome is consistent with the gain in $\mathrm{Li} / \mathrm{LiMn}_{2} \mathrm{O}_{4}$ battery performance resulting from the same change in electrodes, indicating that measurements of microcathodes are relevant to battery device performance. Similar electrochemical measurements were recorded using carbon anodes in the $\mathrm{LiPF}_{6}$ electrolyte solution. Spherical mesocarbon microbeads (MCMB) remained at potentials below $0.14 \mathrm{~V}$ for $20 \mathrm{~h}$ after full charging.
Following those measurements, potential versus time curves were recorded for assembled $\mathrm{LiMn}_{2} \mathrm{O}_{4} / \mathrm{MCMB}$ microbattery structures. The device potential dropped from $4.2 \mathrm{~V}$ to $3.95 \mathrm{~V}$ after just a few minutes, but then remained steady for $\sim 8 \mathrm{~h}$. At that time, the voltage dropped linearly for about $3 \mathrm{~h}$, after which it decreased very rapidly, signaling the end of the battery's lifetime. According to the researchers, the shortcomings in microbattery performance relative to the individual microelectrodes may occur because the nearby cathode material hastens the discharge of the anode and, consequently, the entire device. Nonetheless, this work illustrates the feasibility of using single-particle microbattery structures to determine the factors that limit performance of Li battery devices.

ANDY FRANCIS

\section{Damage to DNA Induced by CdSe/ZnS Core-Shell QDs}

Semiconductor quantum dots (QDs) are a class of nanomaterials being considered for use as fluorescent markers in biological applications. However, before they are used in vivo, researchers want to understand the potential risks. After studying the interactions between watersoluble core-shell quantum dots (cadmium selenide capped by zinc sulfide) and supercoiled, double strands of DNA, researchers M. Green of King's College in London and E. Howman of the University of Oxford uncovered damaged DNA. Their study, which was published in the January issue of Chemical Communications (p. 121; DOI: 10.1039/b413175d), suggests that there may be serious issues to address concerning the use of II-VI semiconductor quantum dots in in vivo applications.

The materials used in the study were commercially available CdSe/ZnS quantum dots with biotin surface functionality. The quantum dots were incubated with the DNA samples in the dark and under UV excitation. After the period of incubation, the DNA was precipitated from the quantum dot solution and run on an electrophoresis gel. This allows detection of supercoiled and uncoiled (damaged) DNA. Samples of DNA that were incubated with a known nicking agent (titania) were also run to determine the position of the damaged DNA strand.

Green and Howman found that DNA damage occurred for samples incubated with quantum dots both under UV excitation and in the dark. Since DNA damage was seen under UV excitation and in the dark, the researchers said that a sulfurrelated free-radical species associated with oxidation of the $\mathrm{ZnS}$ capping layer may contribute to the DNA degradation. According to Green and Howman, the slow oxidation and gradual desorption of surface oxide into solution can explain why DNA is damaged under the studied conditions. Results on assays in which the dots were isolated from the DNA immediately after exposure suggest that the DNA is modified immediately upon exposure to the semiconducting material. The researchers suggest that development of core-shell structures in which the charge is completely confined to the core (e.g., through the use of doping or alternate core-shell materials) may avoid the type of damage observed in these studies.

JEREMIAH ABIADE

\section{Gamma Irradiation Enables Template-Free Synthesis of Polyaniline Nanofibers and Nanorods}

Polyaniline nanofibers are attracting considerable attention for possible applications in electronic devices, especially sensors. They may also be useful as onedimensional electrical conductors and nanoscale electrical connections. Generally, polyaniline nanofibers are made using complicated procedures that require templates. A group of researchers from the University of Missouri-Rolla, led by M.F. Bertino from the Department of Physics and including S.K. Pillalamarri and F.D. Blum from the Department of Chemistry, A.T. Tokuhiro from the Department of Nuclear Engineering, and J.G. Story from the Department of Physics, designed a procedure for polyaniline nanofiber fabrication without using templates. Compared with other "templateless" approaches reported in the past year, this method is probably environmentally friendlier, since it does not require organic solvents, as reported in the January 25 issue of Chemistry of Materials (p. 227; DOI: $10.1021 / \mathrm{cm} 0488478$ ). Gamma rays were employed to irradiate aqueous solutions, which include aniline, ammonium peroxydisulfate (APS), and hydrochloric acid. Surprisingly, the radiation controlled the morphology of the polymer, but did not affect the polymer yield. Transmission electron micrographs showed that the polymer had a nanofiber morphology. Typical nanofiber diameters were 50-100 $\mathrm{nm}$ and their lengths were 1-3 $\mathrm{m}$. X-ray diffraction and Fourier transform infrared spectroscopy gave detailed chemical structure information on the fibers and indicated that the polymer chemical structure was not affected by irradiation.

This template-free technique was also applied in the fabrication of polyaniline rods, the researchers said. When the APS 